\title{
Análise jurídica dos critérios axiológicos de avaliação de medicamentos pela Comissão Nacional de Incorporação de Tecnologias ao SUS - CONITEC
}

Legal analysis of the axiological criteria for evaluation of medicinal products by the National Technology Incorporation Commission - CONITEC

Análisis jurídicos de los criterios axiológicos para la evaluación de los medicamentos por la Comisión Nacional de Tecnología - CONITEC

Álvaro Luis Araújo Sales Ciarlini ${ }^{1}$

RESUMO: A Lei no 12.401, de 28 de abril de 2011 estabeleceu um sofisticado sistema axiológico de avaliação das demandas pela incorporação de medicamentos ao Sistema Único de Saúde utilizados pela Comissão Nacional de Incorporação de Tecnologias. A aplicação desses critérios de valor deve ser devidamente investigada pelo Juiz, sempre que a pretensão em obter medicamento não incorporado ao rol dos fármacos disponibilizados pelo sistema for submetida ao crivo judicial, possibilitando-se a determinação de estratégias argumentativas que podem ser utilizadas nas sentenças judiciais no âmbito das ações individuais cominatórias.

Palavras-chave: Avaliação. Incorporação. Medicamento. Sistema Único de Saúde.

ABSTRACT: Law no 12.401, of April 28, 2011 established a sophisticated system of axiological assessment of demands for the incorporation of drugs into the health public system, which will be used by the National Committee for Incorporation of Technologies. Judge should properly investigate the application of these criteria value, whenever the claim in unincorporated get medicine to the list of drugs available through health public system is subjected to judicial scrutiny, allowing the determination of argumentative strategies that can be used in court rulings within individual lawsuit.

Keywords: Evaluation. Incorporation. Medicine. Unified Health Public System.

RESUMEN: La Ley $n^{\circ} 12.401$, de 28 de abril, 2011 estableció un sofisticado sistema de evaluación axiológica de las acciones judiciales para la incorporación de medicamentos para el Sistema Público de Salud utilizado por el Comité Nacional de Incorporación de Tecnología. La aplicación de estos criterios de valor debe estar debidamente investigado por el juez siempre que la pretensión de obtener la droga no integradas en la lista de medicamentos proporcionados por el sistema se somete al escrutinio judicial, lo que permite la determinación de las estrategias argumentativas que se puede utilizar en las sentencias judiciales dentro de las acciones individuales.

Palabras-llave: Evaluación. La incorporación. Medicina. Sistema Único de Salud.

\footnotetext{
${ }_{1}$ Magistrado do Tribunal de Justiça do Distrito Federal e Territórios. Mestre e Doutor em Filosofia do Direito pela Universidade de Brasília-UnB. Email: alvaro.ciarlini@uol.com.br
} 


\section{Introdução}

O presente artigo tem por objetivo avaliar os critérios axiológicos delineados na Lei № 12.401, de 28 de abril de 2011 (1), quando de sua utilização pela Comissão Nacional de Incorporação de Tecnologias ao SUS - CONITEC. Essa análise pretende situar o tema sob a ótica da judicialização da pretensão em obter medicamento não incorporado ao rol dos fármacos disponibilizados pelo sistema público de saúde, para dar destaque às possíveis estratégias argumentativas que podem ser utilizadas nas sentenças judiciais no âmbito das ações individuais cominatórias.

Com a finalidade de dar mais densidade ao exame desse tópico, procedendo-se ao devido estudo, inclusive, do conceito de integralidade previsto no sistema jurídico pátrio, serão avaliados os argumentos constantes nos Relatórios de Recomendação expedidos pela CONITEC, diante do pedido de incorporação do medicamento que tem como princípio ativo o Fingolimode, cujo nome comercial é Gilenia ${ }^{\mathrm{TM}}$.

Inicialmente, serão examinados os argumentos que constam no Relatório de Recomendação da Comissão Nacional de Incorporação de Tecnologias no SUS CONITEC, de 4 , de julho de 2012, intitulado 'Fingolimode para o tratamento da esclerose múltipla' (2).

Diante da análise do caso de modo circunstanciado, com embasamento nos requisitos delineados no art. 19-R, $\S 2^{\circ}$, da Lei oㅜ 12.016/2014, a CONITEC deliberou por "não recomendar a incorporação do medicamento Fingolimode para tratamento da Esclerose Múltipla", tendo sido essa proposição devidamente acolhida pelo Secretário de Ciência, Tecnologia e Insumos Estratégicos do Ministério da Saúde, por meio da Portaria SCTIE/MS № 25, de 13 de setembro de 2012 (3).

Nos casos diariamente submetidos ao exame do Poder Judiciário (4), no entanto, o pedido de concessão desse medicamento, pelo SUS, a despeito da já mencionada negativa de incorporação pelo Ministério da Saúde, é procedido por meio de ações cominatórias individuais (5) sob a alegação de que esses pacientes já cumpriram todos os estágios terapêuticos preconizados no Protocolo do Ministério da Saúde para a referida doença. Os pacientes queixam-se ainda de que não podem mais se beneficiar 
com o uso dos medicamentos disponibilizados pelo sistema púbico de saúde, pois para eles a última estratégia terapêutica deve consistir no uso do Fingolimode.

As defesas judiciais usualmente oferecidas pela Administração Pública ressaltam que o medicamento Fingolimode foi inicialmente rejeitado pelo Ministério da Saúde, pois seu uso apresenta resultados limitados quanto ao quesito 'segurança'. Dizem também que existem tratamentos padronizados, já constantes nas políticas públicas de saúde, nos estritos termos dos artigos 196 e 198 da Constituição Federal (6) e da Lei no 8080, de 19 de setembro de 1990 (7).

Por isso, pretende-se saber se um medicamento que não foi incorporado para tratamento dos pacientes do Sistema Único de Saúde poderia ser objeto de tutela jurisdicional positiva. Com efeito, é importante ressaltar ainda que existe protocolo clínico para a Esclerose Múltipla no Ministério da Saúde, a recomendar outros tratamentos já 'no mercado' há mais tempo, e que o tratamento é de alto custo (2).

O problema consiste em indagar se o conceito de integralidade previsto na Lei $\mathrm{n}^{\circ}$ 12.401/2011 estaria a abranger todo e qualquer tipo de tratamento, ou medicamento, ainda que experimental, ou seja, se não deveria haver restrições ao acesso a certos medicamentos, aqueles experimentais e sem registro no Brasil, ou mesmo sem 'comprovação científica' de eficácia terapêutica, ou, como no caso do Fingolimode, desprovidos de recomendação positiva, expedida pela CONITEC, para sua incorporação ao SUS, bem como da devida aprovação pelo Secretário de Ciência, Tecnologia e Insumos Estratégicos do Ministério da Saúde.

Para avaliar corretamente essa questão, observe-se que foi engendrada, a partir do ano de 2004, no âmbito próprio do SUS, a Política Nacional de Assistência Farmacêutica, aprovada pelo Conselho Nacional de Saúde, como figura claramente na Resolução no 338, de 6 de maio de 2004 (8). Essa política pública está fundamentada basicamente nos três pilares já anteriormente fixados na Política Nacional de Medicamentos, revelada pela Portaria № 3916, de 30 de outubro de 1998 (9), sendo eles: 1) garantia da segurança, eficácia e qualidade dos medicamentos; 2) promoção do seu uso racional; e 3) acesso da população aos medicamentos considerados essenciais, previstos na Relação Nacional de Medicamentos Essenciais - RENAME. 
É importante ressaltar que faz parte da política nacional de dispensação de medicamentos do SUS adotar um rol padronizado de fármacos, com o objetivo de ofertar esses produtos regularmente aos utentes do sistema, diante do grande número, no mercado farmacêutico, de medicamentos que têm os mesmos princípios ativos, com indicações terapêuticas análogas.

Por isso, o SUS deve promover uma seleção de medicamentos ofertados pelo mercado com a utilização de critérios de razoável cientificidade, visando à segurança e eficácia no tratamento dispensado aos utentes do sistema.

Tal escolha é plenamente justificável, pois, ao alterar a Lei nº 8080/1990, a Lei nº 12.401/2011 pretendeu estabelecer requisitos juridicamente aceitáveis para a integralidade ${ }^{2}$. Ou seja, esse atributo será plenamente atendido quando o medicamento previsto estiver em protocolo clínico ou diretriz terapêutica, ou mesmo, em sua falta, com base nas relações de medicamentos instituídas pelo gestor federal do SUS, ou ainda, de forma suplementar, com respaldo nas listas de medicamentos dos gestores estaduais ou municipais do Sistema.

Para que os protocolos e diretrizes instituídas pelo Poder Público contemplem efetivamente a melhor tecnologia e orientem-se pelos critérios racionais de dispensação de medicamentos, no intuito de conferir a máxima efetividade ao art. 196 da

2 Art. 19-N. Para os efeitos do disposto no art. 19-M, são adotadas as seguintes definições:

(...)

II - Protocolo clínico e diretriz terapêutica: documento que estabelece critérios para o diagnóstico da doença ou do agravo à saúde; o tratamento preconizado, com os medicamentos e demais produtos apropriados, quando couber; as posologias recomendadas; os mecanismos de controle clínico; e o acompanhamento e a verificação dos resultados terapêuticos, a serem seguidos pelos gestores do SUS."

"Art. 19-O. Os protocolos clínicos e as diretrizes terapêuticas deverão estabelecer os medicamentos ou produtos necessários nas diferentes fases evolutivas da doença ou do agravo à saúde de que tratam, bem como aqueles indicados em casos de perda de eficácia e de surgimento de intolerância ou reação adversa relevante, provocadas pelo medicamento, produto ou procedimento de primeira escolha.

Parágrafo único. Em qualquer caso, os medicamentos ou produtos de que trata o caput deste artigo serão aqueles avaliados quanto à sua eficácia, segurança, efetividade e custo-efetividade para as diferentes fases evolutivas da doença ou do agravo à saúde de que trata o protocolo."

"Art. 19-Q. A incorporação, a exclusão ou a alteração pelo SUS de novos medicamentos, produtos e procedimentos, bem como a constituição ou a alteração de protocolo clínico ou de diretriz terapêutica, são atribuições do Ministério da Saúde, assessorado pela Comissão Nacional de Incorporação de Tecnologias no SUS.

$\S 1^{\circ}$ A Comissão Nacional de Incorporação de Tecnologias no SUS, cuja composição e regimento são definidos em regulamento, contará com a participação de 1 (um) representante indicado pelo Conselho Nacional de Saúde e de 1 (um) representante, especialista na área, indicado pelo Conselho Federal de Medicina.

§ 2ํㅡ O relatório da Comissão Nacional de Incorporação de Tecnologias no SUS levará em consideração, necessariamente:

I - As evidências científicas sobre a eficácia, a acurácia, a efetividade e a segurança do medicamento, produto ou procedimento objeto do processo, acatadas pelo órgão competente para o registro ou a autorização de uso;

II - A avaliação econômica comparativa dos benefícios e dos custos em relação às tecnologias já incorporadas, inclusive no que se refere aos atendimentos domiciliar, ambulatorial ou hospitalar, quando cabível. " 
Constituição Federal, o art. 19-P da Lei ํㅜ 8.080/1990 previu a submissão da matéria à Comissão Nacional de Incorporação de Tecnologias (CONITEC), tema devidamente regulamentado pelo Decreto № 7.646, de 21 de dezembro de 2011 (10), que se encontra louvado nos seguintes critérios: a) análise das evidências científicas a respeito da eficácia, efetividade e segurança do medicamento, produto ou procedimento, devidamente acatadas pelo órgão competente para o registro ou a autorização de uso; e b) avaliação econômica comparativa dos benefícios e dos custos em relação às tecnologias já incorporadas, inclusive no que se refere aos atendimentos domiciliar, ambulatorial ou hospitalar, quando cabível. ${ }^{3}$

Com o objetivo de obstar a omissão ou o descompasso dos protocolos, diretrizes e listas, em relação aos produtos ofertados pelo mercado farmacêutico, foi fixado o prazo de 180 dias para o exame da matéria pela CONITEC, nos moldes do art. 19-R da mesma lei, contados da data em que foi protocolado o pedido pelo interessado na

${ }^{3} \mathrm{O}$ art. $4^{\circ}$ do Decreto $n^{\circ}$ 7.646/2011 assim dispõe:

Art. $4^{\circ}$ À CONITEC compete:

I - Emitir relatório sobre:

a) a incorporação, exclusão ou alteração pelo SUS de tecnologias em saúde; e

b) a constituição ou alteração de protocolos clínicos e diretrizes terapêuticas; e

II - propor a atualização da Relação Nacional de Medicamentos Essenciais - RENAME nos termos do art. 25 do Decreto no 7.508, de 28 de junho de 2011.

Parágrafo único. Para o cumprimento de suas competências a CONITEC poderá:

I - Solicitar às unidades do Ministério da Saúde:

a) a elaboração de proposta de constituição ou de alteração de protocolos clínicos e diretrizes terapêuticas de interesse para o SUS;

b) a realização de avaliação das solicitações de incorporação, alteração ou exclusão de tecnologias no âmbito do SUS; e

c) estudos de impacto orçamentário no SUS em virtude da incorporação, exclusão ou alteração de tecnologias em saúde pelo SUS;

II - Solicitar à Secretaria de Ciência, Tecnologia e Insumos Estratégicos do Ministério da Saúde:

a) a realização e contratação de pesquisas e estudos;

b) a celebração de acordos de cooperação técnica com entidades públicas e privadas e hospitais de ensino para a realização de estudos de avaliação de tecnologias em saúde; e

c) a celebração de acordos de cooperação técnica com órgãos e entidades públicas estrangeiras com atribuições afins;

III - solicitar às unidades do Ministério da Saúde e às entidades a ele vinculadas informações relativas ao monitoramento de tecnologias em saúde;

IV - Solicitar informações à Agência Nacional de Vigilância Sanitária - ANVISA relativas ao registro, indicações, características, monitoramento de mercado e vigilância pós-comercialização de tecnologias em saúde, além de outras informações necessárias;

V - Solicitar informações à Câmara de Regulação do Mercado de Medicamentos - CMED, criada pela Lei no 10.742, de 6 de outubro de 2003;

VI - Disponibilizar informações a órgãos e entidades públicas para gestão de tecnologias em saúde, ressalvadas as hipóteses de sigilo previstas em Lei;

VII - organizar repositório de informações sobre tecnologias em saúde; e

VIII - constituir subcomissões técnicas no âmbito da CONITEC."

${ }^{3}$ Ministério da Saúde. Resolução no 338, de 6 de maio de 2004, DOU, Seção 1 n. 96, 20 de maio de 2004. 
incorporação, exclusão e alteração de listas e protocolos clínicos e diretrizes terapêuticas ( $\S 1^{\circ}$, inciso I), a partir da instauração do procedimento administrativo aludido, com a possibilidade de prorrogação por 90 (noventa) dias corridos, quando devidamente justificada pelas circunstâncias peculiares de cada caso.

Percebe-se, então, que a incorporação de novas tecnologias ou novos fármacos, ao SUS, deve resultar de procedimento administrativo dotado de duas fases, sendo a primeira delas a análise técnica adequada, ou seja, a devida consideração da Administração Pública a respeito das evidências científicas comprobatórias da eficácia, acurácia, efetividade e segurança do fármaco, produto ou procedimento em exame. A segunda consiste na avaliação econômica comparativa dos benefícios e dos custos em relação às tecnologias já incorporadas, inclusive quanto aos atendimentos domiciliar, ambulatorial ou hospitalar, quando essas providências mostrarem-se cabíveis (art. 19-Q, $\S 2^{\circ}$, da Lei $n^{\circ}$ 8.080/1990) (8)

Em síntese, pode-se dizer que os preceitos da universalidade e da integralidade das ações de saúde no âmbito do SUS estão respaldados: a) no melhor conhecimento técnico-científico disponível; b) na proteção do cidadão nas ações de assistência, prevenção e promoção à saúde por meio de processo seguro de incorporação de tecnologias pelo SUS; c) na incorporação de tecnologias por critérios racionais e parâmetros de eficácia, eficiência e efetividade adequados às necessidades de saúde; e d) na incorporação de tecnologias que sejam relevantes para o cidadão e para o sistema de saúde, baseada na relação custo-efetividade.

No caso do Fingolimode, o conteúdo do Relatório $n^{\circ}$ 4, de julho de 2012, da CONITEC, inicialmente decidiu, em linhas gerais, "não incorporar o medicamento fingolimod para tratamento de Esclerose Múltipla no Sistema Único de Saúde (SUS)"(11), a despeito da evidência de que o medicamento apresentara resultado terapêutico favorável no caso da esclerose múltipla. A justificativa para tanto fundamentou-se no requisito da segurança no uso do fármaco, a demandar a adoção de precauções a respeito do tema.

A situação ora descrita revela-se sobremaneira paradoxal, pois a despeito do ganho em racionalidade representado pelas recente alteração procedida no texto da Lei no 8080/1990, não se pode vislumbrar a necessária coerência entre o contexto 
normativo dos artigos 196 e 198 da Constituição, ao afirmar o direito à saúde, e o resultado da primeira deliberação procedida pela CONITEC quanto aos critérios de eficácia, acurácia, efetividade e segurança do fármaco, produto ou procedimento em exame, bem como a avaliação econômica comparativa dos benefícios e dos custos em relação ao seu uso.

Ora, essa assimetria deve-se à diversidade de critérios utilizados a) pelo Administrador Público, para o fim de justificar a propalada racionalidade na equação formada pelos dados orientadores dos meios e fins pretendidos; e b) pelo Juiz, que pretende proferir uma decisão que reconheça, ou não, a existência do direito subjetivo de um paciente ao recebimento de medicação pelo sistema público de saúde. Ambas as decisões devem partir do pressuposto, já aludido, de que o medicamento Fingolimode apresentou satisfatório resultado terapêutico, nas hipóteses descritas no relatório da CONITEC, mas também devem considerar que há incertezas quanto à segurança de seu uso.

Ora, a ratio administrativa adotada na fundamentação constante no relatório, por imperativo legal, deve estar embasada nos seguintes critérios axiológicos: a) eficácia, que é a soma dos efeitos terapêuticos do medicamento ou da tecnologia a ser incorporada; b) acurácia, que é o termo relacional que visa a medir a exatidão do efeito clínico produzido pelo medicamento ou tecnologia (portanto, de sua eficácia), em face do fim terapêutico pretendido; c) efetividade, que designa a medida que tem como pressuposta uma equação entre a eficácia, a acurácia e o resultado terapêutico esperado; e finalmente a d) segurança, que consubstancia uma equação do tipo custo/benefício, tendo por pressuposta a efetividade do tratamento, em relação aos riscos potencialmente produzidos pelo medicamento à saúde do paciente.

Estamos diante de elementos axiológicos cooriginários, correspectivos e complementares, todos indicadores de parâmetros de justificação da escolha apta a realizar a pretendida integralidade do tratamento disponibilizado pelo SUS. Cuida-se de um sistema de proposições que têm por objetivo definir, em seu conjunto, a máxima utilidade e potencialidade do tratamento financiado com recursos públicos, diante de elementos balizadores fundados em uma equação do tipo custo/benefício. Por isso mesmo, pretende-se conceder aos utentes do sistema um conjunto de serviços, 
insumos, tecnologias e medicamentos aptos à finalidade curativa buscada, mas, ainda assim, submetido a critérios que apontem para o alvo da correção e da racionalidade, portanto, do equilíbrio e da razoabilidade.

Podemos, portanto, ver a integralidade como atributo qualificador do atendimento, a partir de um conjunto de haveres, bens e serviços, tal qual previsto no art. 198, inc. II, da Constituição da República ${ }^{4}$ (6) apto a designar o que é suficiente e necessário ao atendimento dos utentes desse sistema que, sob o signo da universalidade, pretende ser amplo e pleno, e também justo, pois todos temos "direito à saúde" 5 . Ainda assim, esse atendimento mostra-se limitado a certos e determinados critérios próprios a sua concessão, justamente os definidos pelas políticas públicas levadas a efeito pelo SUS.

Sem dúvida, isso decorre translucidamente das normas constitucionais mencionadas e define o próprio âmbito do direito fundamental à saúde que, no tópico alusivo à incorporação de medicamentos e tecnologias ao SUS, está submetido a critérios valorativos específicos, deliberados no âmbito de uma comissão legal e constitucionalmente legitimada para esse fim.

O que, então, terá restado ao trabalho jurisdicional? Para avaliar adequadamente a possibilidade de defender a existência de uma situação jurídica designativa do direito à saúde, em face do Estado, nesse caso em especial, convém ressaltar que a ideia de direitos subjetivos, desenvolvida sob a ideologia do liberalismo político clássico, a partir do utilitarismo de Bentham 12) constituiu importante premissa doutrinária adotada por Ronald Dworkin em seu ensaio Rights as Trumps (13). Nessa tese reside a ideia de Dworkin a respeito do igualitarismo liberal, que sustenta um ponto de partida segundo o qual os direitos consubstanciam uma espécie de trunfo, podendo assim ser impostos em uma determinada relação entre o indivíduo e a sociedade.

Sem pretender redefinir o sentido do termo 'ter direitos', Dworkin trata de conciliá-lo com a noção de bem comum, visível na seguinte afirmação: "Rights are best understood as trumps over some background justification for political decisions that states a goal for the community as a whole (13).

\footnotetext{
${ }^{4}$ Art. 196, primeira parte CF/88.

${ }^{5}$ Artigos 196 e 198 CF/88
} 
Para tanto, essa doutrina se opõe ao modelo utilitarista, ao afirmar que o princípio da igualdade pode servir de parâmetro de justificação dos direitos ou do bem-estar geral. Esse posicionamento teórico incompatibiliza-as ainda com o positivismo jurídico, vendo nos trunfos a possibilidade de assegurar aos indivíduos a proteção de certos interesses nascidos em circunstâncias especiais, a fim de lhes garantir padrões mínimos de existência, em homenagem a uma noção mais substancial de igualdade, uma vez que "representa a promessa da maioria às minorias de que sua dignidade e igualdade serão respeitadas" (14).

Estamos justamente diante de um sistema de apreciação que permite dialetizar o que o utente do sistema de saúde pública deseja obter do Estado, ou seja, um determinado medicamento indicado para tratar do insidioso e grave quadro de saúde resultante de seu acometimento pela esclerose múltipla, em face das possíveis justificativas para lhe impor uma restrição à fruição desse peculiar serviço de saúde, constantes nos relatórios da CONITEC. Portanto, podemos sintetizar, a partir do trabalho teórico de Dworkin, que o paciente terá direito subjetivo ao recebimento desse medicamento quando, por alguma razão, convém insistir, não for viável supor a suficiência dessa justificativa que pretende legitimar a restrição a sua incorporação ao SUS, calcada em argumentos de tutela dos interesses comuns da sociedade como, por exemplo, na defesa da isonomia de tratamento dos utentes do sistema e na correlata racionalidade dos gastos, em face dos resultados a serem aplicados à generalidade dos pacientes.

Por isso mesmo, muito embora seja inegável o ganho de racionalidade na deliberação a respeito da incorporação de medicamentos ao SUS por meio de um procedimento orientado por sofisticados e incontestáveis critérios axiológicos, é necessário examinar se os elementos de valoração utilizados pela CONITEC são subsistentes quando aplicados a uma situação jurídica concreta.

$\mathrm{Na}$ hipótese do Fingolimode, a análise tópica das situações expostas permite afirmar que o relatório CONITEC - 4, de julho de 2012, trouxe ainda mais complexidade ao exame da matéria, mostrando-se carente de argumentos de justificação a respeito da restrição imposta. Ora, a par de se encontrar louvado em um modelo de análise obtido por meio de incontestável e legítimo procedimento público e plural, provido de inegável 
racionalidade, a preponderância dada ao argumento do risco à saúde dos pacientes que já se submeteram, sem sucesso, a outros esquemas terapêuticos para o controle da esclerose múltipla, parece decorrer da inadequada avaliação dos elementos de valor e de peso, descritos como seus critérios axiológicos, notadamente a respeito da dialeticidade entre a efetividade e a segurança do medicamento, diante do natural risco imposto aos pacientes, vitimados que estão por uma doença neurológica crônica, irreversível e progressiva.

Note-se que os possíveis efeitos adversos cardiovasculares descritos no referido relatório, alusivos à primeira dose do medicamento, poderiam bem ter sido objeto de controle rigoroso durante as primeiras horas após a administração do medicamento, com a devida preparação do local para atendimento de urgência.

Logo, se não se encontrava devidamente esclarecida e justificada a restrição imposta à concessão desse medicamento em particular, deve-se afirmar, em contrapartida, diante dessa linha de fundamentação, que subsistiria o direito subjetivo público do paciente em obter resposta jurisdicional positiva do Estado para o recebimento do Fingolimode, em face da restrição imposta pelo Relatório CONITEC - 4, de julho de 2012.

Feitas essas ponderações, resta examinar as considerações finais do Relatório de Recomendação da CONITEC - 113, de abril de 2014 (11).

O mencionado estudo propôs recomendação ${ }^{6}$ para a utilização de dois outros medicamentos, quais sejam, a Betainterferona ou o Glatirâmer para a primeira e a segunda linhas de tratamento, permanecendo o Natalizumabe e o Fingolimode para a terceira linha terapêutica, com primazia para o uso do primeiro, principalmente em virtude da maior experiência em sua utilização, e ainda, em decorrência do constatado problema alusivo à segurança, relatado ao uso do Fingolimode. Por isso, em sua deliberação procedida na 23를 Reunião da CONITEC, realizada aos 12 de março de 2014, houve a recomendação da incorporação do Fingolimode para pacientes com

\footnotetext{
${ }^{6}$ Esclareça-se que a Portaria/MS $\mathrm{n} \cong 23$, de 27 de junho de 2014 , tornou pública a decisão de "não incorporar o Fingolimode para a primeira e segunda linhas do tratamento da esclerose múltipla no âmbito do Sistema Único de Saúde - SUS", enquanto que a Portaria/MS no 24, de 27 de junho de 2014, consolidou "a decisão de incorporar o Fingolimode no sistema único de saúde nos casos de: pacientes com esclerose múltipla remitente-recorrente; com surtos incapacitantes após falha ao uso de betainterferona e de glatirâmer; com impossibilidade de uso de natalizumabe e sem contraindicação ao uso de Fingolimode conforme protocolo clínico e diretrizes terapêticas".
} 
esclerose múltipla remitente-recorrente, com surtos incapacitantes, a despeito da subministração da Betainterferona e do Glatirâmer, esses em primeira e segunda linhas de tratamento, desde que, diante de relatório clínico devidamente justificado, não seja possível o uso do Natalizumabe e não exista contraindicação do uso do Fingolimode.

Em face dessas considerações, é curial também que a incorporação do Fingolimode deverá estar "condicionada à disponibilização em centros especializados, com infraestrutura adequada ao monitoramento dos pacientes que utilizarem 0 medicamento" (11).

O Relatório de Recomendação da CONITEC - 113, de abril de 2014, trouxe inegavelmente mais legitimidade à análise pragmática dos elementos axiológicos em exame, pois previu um critério progressivo para a concessão desse medicamento pelo Poder Público, tratando de agregar, aos argumentos utilizados, alguns elementos de justificação ancorados em tópicos reveladores da 'cientificidade' das escolhas indicadas pelo aludido estudo.

Eis um problema de grande envergadura epistemológica: as críticas usualmente dirigidas contra a 'cientificidade' de alguns métodos de trabalho pretensamente científicos consistem precisamente na ausência de cadeias dedutivas necessárias entre os argumentos explicativos de uma determinada construção teórica e os dados obtidos a partir da observação empírica, sendo comum a fundamentação respaldada em elementos retóricos a respeito da cientificidade de uma dada proposição técnica, sem, portanto, a devida preocupação com os respectivos critérios indutivos ou dedutivos que permitam a aferição mínima dos pretensos resultados da pesquisa (15), a partir da justificável correlação entre a teoria e o experimento. Isso invariavelmente transforma os resultados pretensamente científicos em dados meramente arbitrários (16)

Por isso, pode-se perguntar, no âmbito da epistemologia, sobre a possibilidade de estabelecer um conhecimento teórico (16) minimamente confiável que possa subsidiar a necessária e consistente base científica a respeito desse tema. As questões em exame dizem respeito à explicação e fundamentação científicas (16) e certamente devem conduzir ao estabelecimento de critérios metodológicos de demarcação de um sistema científico. No entanto, o que caracteriza a ciência, distinguindo-a da pseudociência, para 
Karl Popper, não é a estrutura de seus produtos acabados, mas o elenco justificável e validável de seus métodos (17).

Assim, em tese, a afirmação a respeito da 'cientificidade' de uma dada proposição sobre a eficácia, acurácia ou efetividade dos medicamentos descritos em relatórios da CONITEC não pode ser recebida, efetivamente, como uma 'verdade científica' universal e irrefutável, mas deve ser examinada com justificável cuidado e circunspecção, no caso concreto submetido ao Juiz, inclusive com o auxílio de perícias, ou com o acesso a outros experimentos ou teses em curso na literatura especializada.

Por isso mesmo, a só existência do conteúdo do Relatório de Recomendação da CONITEC - 113, de abril de 2014, a priori, não afasta as perguntas a respeito do direito fundamental do paciente, pois não pode nos conceder a exata dimensão da eventual legitimidade dos argumentos aptos a funcionar como justificativa suficiente para a restrição imposta aos pacientes acometidos por esclerose múltipla.

Além disso, diante da apreciação dos critérios de suficiência e propriedade dos requisitos de eficácia, eficiência, acurácia, efetividade e segurança, ora descritos como requisitos axiológicos imprescindíveis ao exame a ser procedido pela CONITEC, haverá ainda ensejo para o ajuizamento de ação que trate da tutela aos interesses metaindividuais dos pacientes, pois a via jurisdicional individual não pode ser o palco adequado para o exame desse tema em particular, que tem repercussões metaindividuais (18). Essa matéria, evidentemente, deverá ser inexoravelmente submetida ao crivo jurisdicional por intermédio da Ação Civil Pública, palco adequado para o aprofundamento discursivo jurídico e lógico-teórico de tema de tamanha densidade constitucional, a envolver o direito fundamental social à prestação, pelo Estado, de um específico serviço de saúde, tema que deve ser tratado diante de suas complexas peculiaridades (19), inclusive com a necessária preservação do princípio isonômico previsto no Texto Constitucional.

À guisa de conclusão, é viável supor, à luz dos argumentos de Ronald Dworkin, a possibilidade da intervenção judicial com o escopo de obter a tutela da esfera jurídica dos pacientes já submetidos ao tratamento usualmente fornecido pelo SUS, para doenças crônicas e progressivas, como é o caso da esclerose múltipla, tendo havido, ou não, o cumprimento integral dos estágios terapêuticos estabelecidos pelo Ministério da 
Saúde. Isso se mostra justificável desde que não tenham sido constatados os resultados favoráveis esperados, no tempo e modo suficientes e necessários para lidar eficientemente com esses agravos de saúde, a critério dos dados técnicos e científicos aptos à justificação dessa providência médica.

Finalmente, no exame do caso concreto submetido à apreciação judicial, portanto, à vista da avaliação circunstanciada dos referidos elementos técnicos e científicos, o juiz deverá conceder a tutela jurisdicional individual específica buscada pela parte ${ }^{7}$ afirmando o direito fundamental à saúde do paciente, desde que, em nome do princípio da integralidade, e, louvado no critério do direito como trunfo, não se convença da legitimidade dos argumentos expostos como justificativas suficientes para a restrição imposta, pela CONITEC, ao tratamento em questão.

${ }^{7}$ Art. 461, caput, do CPC (art. 497, caput, do NCPC). 


\section{Referências}

1 Brasil. Lei no 12.401, de 28 de abril de 2011. Altera a Lei no 8.080, de 19 de setembro de 1990, para dispor sobre a assistência terapêutica e a incorporação de tecnologia em saúde no âmbito do Sistema Único de Saúde - SUS. [Acesso em 19.fev.2015].

Disponível em http://www.planalto.gov.br/ccivil_03/_Ato2011-2014/2011/Lei/L12401.htm

2 Brasil. Ministério da Saúde. Relatório de Recomendação da Comissão Nacional de Incorporação de Tecnologias no SUS - CONITEC de 4 de julho de 2012 [Acesso em 22.out.2013]. Disponível em: www.saude.gov.br.

3 Brasil. Ministério da Saúde. Portaria SCTIE/MS no 25, de 13 de setembro de 2012. Brasília: Diário Oficial da União, no 179, de 14 de setembro de 2012, p. 73.

4 Diniz, D; Machado, TRC; Penalva, J. A judicialização da Saúde no Distrito Federal, Brasil. Ciência \& Saúde Coletiva, 19(2):591-598, 2014, p. 591-598. DOI: 10.1590/141381232014192.23072012

5 Tribunal de Justiça do Distrito Federal e Territórios (TJDFT). Acórdão no 803766, 20130110005702APC. Relator: Alfeu Machado, Revisor: Leila Arlanch. 1aㅡ Turma Cível, Data de Julgamento: 17/07/2014. Brasília: Diário da Justiça (DJE), DE 22 de julho de 2014, p. 68.

6 Brasil. Constituição da República Federativa do Brasil. Brasília: Senado Federal, 2011

7 Brasil. Lei 8080 de 19 de setembro de 1990. Dispõe sobre as condições para a promoção, proteção e recuperação da saúde, a organização e o funcionamento dos serviços correspondentes e dá outras providências. [ Acesso em 19.fev.2015].

Disponível em: http://www.planalto.gov.br/ccivil_03/Leis/L8080.htm

8 Brasil. Ministério da Saúde. Resolução n 338 de 6 de maio de 2004 do Conselho Nacional de Saúde. Brasília: Diário Oficial da União, Seção 1, n. 96, de 20 de maio de 2004.

9 Brasil. Ministério da Saúde. Portaria MS no 3.916, de 30 de outubro de 1998. [Acesso em 22.out.2013]. Disponível em: www.saude.gov.br.

10 Brasil. Decreto no 7.646/2011 de 21 de dezembro de 2011.

11 Brasil. Ministério da Saúde. Relatório de Recomendação da Comissão Nacional de Incorporação de Tecnologias no SUS (CONITEC) de 4 de julho de 2012. [Acesso em 22.out.2013]. Disponível em: www.saude.gov.br/conitec

12 Bentham, J; Mill, JS. The Classical Utilitarians - Benthan and Mill. Indianápolis: Hackett, 1979.

13 Dworkin, R. Rights as Trumps? In: Waldron, J. Theory of rights. Oxford: University Press, 1984, p. 153-167. 
14 Dworkin, R. Levando os direitos a sério. Trad. Nélson Boeira. São Paulo: Martins Fontes, 2002, p. XV.

15 Nikles, T. Methodology, heuristics and racionality; J. C. Pitt and M. Pera (eds.). Rational Changes in Science. Dordrecht: D. Reidel Publishing Company, 1987, p. 103132.

16 Feyerebend, P. Contra o método. Octanny S. da Mota e Leônidas Hegenberg (trad). 2 ed. Rio de Janeiro: Francisco Alves, 1977, p. 45-56.

15 Lakatos, I. A crítica e o desenvolvimento do conhecimento. São Paulo: Cultrix, 1979, p. $109-119$.

17 Popper, Karl. A lógica da Pesquisa Científica. 8 ed. São Paulo: Cultrix, 1999, p. 27-35

18 Ciarlini, ALAS. Direito à Saúde - Paradigmas procedimentais e substanciais da Constituição. São Paulo: Saraiva, 2013, p. 231-241

19 Delduque, MC; Marques, SB; Ciarlini, ALAS. Judicialização das Políticas de Saúde no Brasil. In: Alves, SMC; Delduque, MC; Dino Neto, N (orgs.) Direito Sanitário em Perspectiva. Brasília: ESMPU, FIOCRUZ, 2013, p. 209-213.

\section{Como citar este artigo:}

Ciarlini ALAS. Análise jurídica dos critérios axiológicos de avaliação de medicamentos pela Comissão Nacional de Incorporação de Tecnologias ao SUS - CONITEC. Revista Cadernos Ibero-Americanos de Direito Sanitário. 2016 jan./mar, 5(1):205-219. 